\title{
The utility of PET/CT in diagnosing fever of unknown origin: The experience of a large tertiary medical center
}

\author{
Hussein Mahagna ${ }^{1}$, Keren Vaknin ${ }^{1}$, Abdulla Watad ${ }^{2}$, Arsalan Abu-Much ${ }^{3}$, Naim \\ Mahroum ${ }^{1}$, Ora Shovman ${ }^{1}$, Jennifer Ben Shimol ${ }^{4}$, Yehuda Shoenfeld ${ }^{5}$, Howard Amital ${ }^{3}$, \\ and Tima Davidson ${ }^{6}$ \\ ${ }^{1}$ Sheba Medical Center \\ 2Department of Medicine 'B', Zabludowicz Center for Autoimmune Diseases, Sheba Medical \\ Center, Tel-Hashomer, Israel, Sackler Faculty of Medicine, Tel-Aviv University, Israel \\ ${ }^{3}$ Affiliation not available \\ ${ }^{4}$ Edith Wolfson Medical Center \\ ${ }^{5}$ Tel-Aviv University \\ ${ }^{6}$ Sheba Medical Center at Tel Hashomer
}

August 28, 2020

\begin{abstract}
Background: Fever of unknown origin (FUO) is a diagnostic challenge. Positron emission tomography with computed tomography $(\mathrm{PET} / \mathrm{CT})$ is expensive and not widely available. We aimed to evaluate the diagnostic yield of PET/CT in the workup of FUO, and identify the patients for whom this test is most useful. Methods: We reviewed the records of all patients admitted to Sheba Medical Center from 2013 to 2017 who were underwent PET/CT for the evaluation of FUO. Results: Of the 128 patients included, PET/CT was helpful in revealing the cause of FUO in 61 (48\%). A final diagnosis was made in 95 (74\%) patients. Of the $128 \mathrm{PET} / \mathrm{CT}$ tests conducted for the workup of FUO, $61(48 \%)$ had true positive results, $25(20 \%)$ false positive results, $24(19 \%)$ true negative results, and 18 (14\%) false negative results. In a multivariate analysis, helpfulness of PET/CT was independently associated with weight loss and low hemoglobin. The calculated test sensitivity was $77 \%$, specificity was $49 \%$, positive predictive value was $71 \%$ and negative predictive value was $57 \%$. Conclusion: PET/CT is valuable in the diagnostic workup of FUO. When used as a complementary test, it was helpful in diagnosing almost half the patients, and especially in those with low hemoglobin and weight loss.
\end{abstract}

\section{What is already known about this topic?}

-PET/CT occupies a varying place within the work-up of fever or unknown origin.

-While PET/CT is an expensive study, in institutions where this study is performed, it often helps to uncover the source of fever of unknown origin.

-PET/CT is most sensitive in uncovering malignant sources of fever.

\section{What does this article add?}

-PET/CT may uncover the source of fever in up to half of cases of fever of unknown origin, where prior work-up was unrevealing.

-Infection was the most common source of fever identified by PET/CT.

-PET/CT was most useful in diagnosing the cause of fever in patients with anemia and weight loss. 


\section{Introduction}

Fever of unknown origin (FUO) is a frequently encountered condition on internal medicine wards. It was first defined in 1961 as a fever greater than $38.3^{\circ} \mathrm{C}\left(101^{\circ} \mathrm{F}\right)$ for 3 weeks or more, whose origin is not identified despite a thorough work-up [1]. The recommended work-up has been modified over the years. While an abundance of studies may aid in the diagnosis, FUO often poses a great challenge to clinicians, and nearly half the patients ultimately do not receive a definitive diagnosis [2-4].

Positron emission tomography with computed tomography (PET/CT) combines regular CT with nuclear imaging based on the enhanced uptake of fluorodeoxyglucose $\left({ }^{18} \mathrm{~F}-\mathrm{FDG}\right)$ by highly metabolic inflammatory and malignant cells. In the last decade, the PET/CT modality has gained widespread popularity. Several prospective and retrospective studies have suggested an important role of PET/CT in the work-up of FUO [5-8]. Nonetheless, much heterogeneity remains regarding the place of PET/CT within the algorithm of recommended studies. Some consider PET/CT a mandatory part of the work-up, while others regard it as a complementary test that may be performed following the use of other imaging modalities such as CT scans. Differences in study methodologies have produced variable results regarding the value of PET.

Given the lack of widespread availability of PET/CT, and its high costs, the population of FUO patients that is expected to gain benefit from this modality needs to be further characterized. In the current study, we assessed the utility of PET/CT in revealing the correct cause of FUO and evaluated for parameters that may increase the likelihood of diagnostic yield in patients with FUO.

\section{Methods}

\section{Study design}

We reviewed the medical records of all the patients who underwent PET/CT scans as part of FUO work-up between January 1, 2013 and January 30, 2018, at the Chaim Sheba Medical Center (Ramat Gan, Israel). With more than 1,900 beds, this is the largest and most comprehensive tertiary medical center in the Middle East.

Imaging data weas obtained from the picture archive and communication system (PACS, Carestream Health 11.0, Rochester, NY) and clinical data was obtained from the computerized medical records within our medical center.

\section{Participants}

The diagnosis of FUO was made according to the classic Petersdorf's criteria, body temperature $>38.3^{\circ} \mathrm{C}$ on several occasions for at least 3 weeks with no diagnosis despite one week of inpatient or outpatient work-up [1]. This work-up included a thorough history and physical examination, a complete blood count, blood chemistry, blood cultures, hepatitis serologies in the case of abnormal liver tests, urinalysis, and a chest radiograph. In those patients who were admitted, the workup also included more extensive inflammatory and infectious serologies, and abdominal ultrasound or CT.

In our facility, PET/CT was not considered a standard part of the initial workup for patients with FUO, and its usage was preserved for those without a diagnosis despite a complete work-up. Study exclusion criteria were age below 18 years, pregnancy, insufficient data from medical records, active solid or hematologic malignancy, neutropenia, nosocomial infections, or HIV carrying status.

\section{FDG-PET/CT imaging technique}

FDG-PET/CT examinations were performed with a combined FDG-PET/CT scanner (Philips Gemini GXL, Philips Medical Systems, Cleveland OH, USA) that includes a 16-detector row helical CT. This enables simultaneous acquisition of up to 45 trans axial PET images with inter-slice spacing of $4 \mathrm{~mm}$ in one bed position; and provides an image from the vertex to the thigh, with about 10 bed positions. The transaxial field of view and pixel size of the PET images reconstructed for fusion were $57.6 \mathrm{~cm}$ and $4 \mathrm{~mm}$, respectively, 
with a matrix size of $144 \mathrm{x} 144$. The technical parameters used for CT imaging were pitch 0.8 , gantry rotation speed $0.5 \mathrm{~s}$ /rotation, $120 \mathrm{kVp}, 250 \mathrm{mAs}, 3 \mathrm{~mm}$ slice thickness, and specific breath-holding instructions [9-11].

After 4-6 h of fasting, patients received an intravenous injection of $370 \mathrm{MBq}$ F-18 FDG. About 75 min later, CT images were obtained from the vertex to the mid-thigh for about $32 \mathrm{~s}$. When intravenous contrast material was used, CT scans were obtained $60 \mathrm{~s}$ after injection of $2 \mathrm{~mL} / \mathrm{kg}$ of non-ionic contrast material (Omnipaque 300; GE Healthcare). An emission PET scan followed in 3D acquisition mode for the same longitudinal coverage, 1.5 min per bed position. CT images were fused with the PET data and were used to generate a map for attenuation correction. PET images were reconstructed using a line of response protocol with CT attenuation correction, and the reconstructed images were generated for review on a computer workstation (Extended Brilliance Workstation, Philips Medical Systems, Cleveland OH, US [9-11].

\section{Image assessment}

An experienced physician with two specializations (nuclear medicine and radiology, TD), with 20 years' experience, reviewed all the cases identified in the current study. FDG uptake in the lesions was measured by standardized uptake values max (SUVmax), which was calculated by manually generating a region of interest over the sites of abnormally increased FDG activity [9-11].

\section{Data collection and review of PET/CT contribution}

For each patient, we collected data on their demographics, medical history, current episode of fever, laboratory tests, and imaging studies. We examined the PET/CT results and reviewed the final diagnosis if it was made within 3 months of discharge. Finally, we evaluated the results of the PET/CT studies and concluded whether the test was contributory to the final diagnosis. This was determined by two internal medicine consultants. When controversies arose regarding the contributory value of a test, a third physician reviewed the data, and a decision was made based on the rule of the majority.

Tests were considered true positives and therefore contributory if they had positive findings that were relevant to the diagnosis. Studies were labelled as true negative if no positive hypermetabolic findings were observed, and no clinical diagnosis was established during 3 month follow-up. True negative tests (negative PET/CT scan, with no diagnosis made within 3 months) were not considered contributory since excluding diagnoses were not considered of value. Tests were called false positive if PET/CT showed hypermetabolic findings, but these findings did not add to the final diagnosis; or if no diagnosis was made at all within 3 months of follow-up. They were considered false negative if no hypermetabolic findings were observed and the diagnosis was made by other means of investigations.

\section{Statistical analysis}

Descriptive statistics were used to characterize the basic features of the data in the study. We compared the features of patients who had contributory tests to those who had non-contributory ones. We used the Chi-square test and Fisher's exact test for categorical variables, and the T-test and Mann Whitney U test for continuous variables, as appropriate. A p-value of less than 0.05 was considered significant in all the tests. Parameters found to be significantly associated with contributory results of PET/CT (true positive) on univariate analysis were analyzed in a multivariate analysis using forward step logistic regression analysis.

\section{Ethical approval}

This single-institution study was approved by the institutional review board of Sheba Medical Center (SMC18-4875). Informed consent was waived by the institutional review board due to the retrospective nature of the study. All methods were performed in accordance with the institution's guidelines and regulations.

\section{Results}

Study populationIn total, 225 patients were identified as having undergone PET/CT scans as part of an FUO workup. Of them, 97 did not meet study inclusion criteria (Fig. 1). Of the 128 patients who met eligibility criteria and were included within the cohort, $80(62.5 \%)$ were males. The mean age of the patients was $59 \pm$ 
20.3 years (range: 18-93). The mean duration of fever (prior to the performance of PET/CT) was 38 days. Twenty-nine $(22.8 \%)$ of the patients were considered immunosuppressed, due to concomitant treatment with corticosteroids (19 patients) or other immunosuppressive drugs, some of whom were also receiving corticosteroids (19 patients), or by having medical conditions that cause immunosuppression. Twenty-two (17\%) patients had implanted prosthetic devices, including vascular implants, pacemakers and heart valves. Table 1 presents the demographic and clinical characteristics of the study population, according to whether $\mathrm{PET} / \mathrm{CT}$ provided contributory results.

Final diagnoses By the completion of the work-up, 95 patients had $(74.2 \%)$ received a final diagnosis. Most (64.2\% of the diagnosed patients and $47.7 \%$ of the study population) were diagnosed with infectious diseases (Table 2). These included pulmonary infections $(n=12)$, endovascular infections $(n=7)$, endocarditis $(\mathrm{n}=7)$, bacteremia $(\mathrm{n}=4)$, and osteomyelitis $(\mathrm{n}=2)$. Twenty-one $(16.4 \%)$ of the patients were diagnosed with inflammatory diseases, including arthritis $(n=3)$, adult onset Still's disease $(n=3)$, and vasculitis $(n=3)$. Malignancies were diagnosed in 12 patients $(9.4 \%)$, nine $(75 \%)$ of which were hematologic, and included lymphoma $(\mathrm{n}=6)$ and leukemia $(\mathrm{n}=3)$. One patient $(0.8 \%)$ was diagnosed with Kikuchi disease. No compatible diagnosis was made for $33(25.8 \%)$ patients at 3 months following admission. The different diagnoses are presented in Table 2. Figures 4-7 show PET/CT scan images of four patients with FUO who were included in the study.

Contribution of PET/CT to FUO workup Overall, 86 (67.2\%) of the 128 included patients had positive findings in PET/CT scans. In $61(47.7 \%)$ patients, the results were deemed true positive for leading to the final diagnosis. The remaining 25 (19.5\%) had false positive results (Fig. 2).

Forty-two (33\%) of the included patients did not have pathological FDG uptake findings on PET/CT. Indeed, twenty-four (18.8\% of the study population and $57.0 \%$ of patients without diagnostic findings on PET/CT) did not have a final diagnosis at the end of the workup or at 3 months follow-up and were thus considered true negative. The remaining 18 (14\%) patients without a pathological FDG uptake on PET/CT were eventually diagnosed using other modalities and were therefore considered false negative (Fig. 2).

Findings yielded a sensitivity of $77.2 \%$, specificity of $49 \%$, positive predictive value of $70.9 \%$, and negative predictive value of $57.1 \%$.

PET/CT contribution according to disease category Of patients with final diagnoses of inflammatory and infectious conditions, the rates of true positive results were $60.0 \%$ and $61.7 \%$, respectively and the rates of false negative results were $30.0 \%$ and $20.0 \%$, respectively. Of the patients who were given a final diagnosis of malignancy, $8.3 \%$ of them had false positive results. None had false negative results (Fig. 3).

Parameters associated with true positive resultsIn a univariate analysis, weight loss, low concentrations of hemoglobin and low levels of transferrin were associated with true positive diagnoses (Table 1). In a multivariate analysis, hemoglobin was inversely associated with contributory PET/CT tests (odds ratio (OR): 0.597, $95 \%$ confidence interval $(\mathrm{CI}) 0.412-0.866, \mathrm{p}=0.006)$. Weight loss, on the other hand, was positively associated with contributory PET/CT tests (OR: 3.605, 95\% CI 1.123-11.576, $\mathrm{p}=0.031$ ). The association of low-level transferrin with a diagnostic PET/CT was not significant: (OR: 0.992, 95\% CI 0.981-1.003, $\mathrm{p}=$ 0.13) (Table 3).

\section{Discussion}

In this retrospective study, PET/CT proved helpful in diagnosing almost half of all patients who underwent scans as part of FUO work-up. The management of patients with FUO has evolved considerably since this entity was initially described. Nevertheless, many patients remain undiagnosed despite extensive testing. Review of the current literature suggests that PET/CT is a useful part of work-up though reports vary widely, citing numbers which range from $42 \%$ to $72 \%$ of cases in which they assisted in the diagnosis [5$8,12-21]$. The variability of results reported can be explained, in part, by differences in the methodologies employed, definition of FUO, the varying work-up algorithms and the fluctuating place of PET/CT within the algorithms, technique and model of the PET scanners, and how different groups define whether PET/CT 
is helpful to establishing the diagnosis. This diversity makes it difficult to compare among the published studies.

In a prospective study by Schönau et al. [8], PET/CT scan was a mandatory part of the work-up of FUO. All patients with FUO who presented to internal medicine wards were included. PET/CT yielded true positive results in $56.7 \%$ of the patients with FUO. In contrast, we included only patients without a diagnosis after a thorough medical workup; $44.0 \%$ of them underwent CT-scans before advancing to a PET/CT scan.

Our rate of false negative results of PET/CT scans, $14.1 \%$, is comparable to rates reported in the literature. Two thirds of these patients were diagnosed with infectious diseases and one third with inflammatory diseases (familial Mediterranean fever, adult-onset Still's disease, polymyalgia rheumatica and temporal arteritis) while none were diagnosed with malignant diseases. Schönau et al. [8] found false negatives in only $5 \%$ of their cohort. In an early study, Gafter-Gvili et al. [7] reported a rate of false negatives of 18\%; of them, $40 \%$ were eventually diagnosed with infectious diseases, and one patient with a malignant disease. This patient was subsequently diagnosed with marginal zone lymphoma based on bone marrow biopsy.

False positive results were found in one fifth of our patients. One patient was diagnosed with lymphoma, eleven were found to have infections, and 2 were diagnosed with inflammatory conditions. In the remaining $44 \%$, no diagnosis was made at the end of the work-up or at 3 month follow-up. Non-specific findings and physiologic uptake were not considered positive results. Our false positive rates match those reported by other studies which range between $9 \%$ and $30 \%$ [3,7,22,23].

Similar to other studies, infectious diseases were the most common cause for FUO in close to half of patients, followed by inflammatory diseases which were identified in $16.4 \%[1,2,7,24]$. PET/CT was helpful in diagnosing just over $60 \%$ of the infectious cases while it guided clinicians toward the correct diagnosis in exactly $60 \%$ of those with inflammatory diseases. Malignant causes of FUO mostly included hematologic malignancies which made up almost three quarters of these cases. For more than $90 \%$ of patients with malignant causes of FUO, the PET/CT test provided true positive results. PET/CT appears to have missed the diagnosis of cancer in only one patient, whose scan showed increase uptake in the lungs and was ultimately diagnosed with T-cell lymphoma through other diagnostic modalities. Accordingly, PET/CT demonstrated highest efficiency in diagnosing malignant causes of FUO.

In our study, weight loss and low hemoglobin levels were independently associated with PET/CT producing true positive results in yielding the cause of FUO. Likewise, Crouzet et al. [5] illustrated an association between low hemoglobin levels and true positive PET/CT scans. In addition, other studies have shown similar associations between true positives and the presence of adenopathy and elevated levels of c-reactive protein (CRP) $[5,6,8]$. We, however, did not find a significant association between true positive results and biomarkers of inflammation such as leukocytosis and elevated CRP. Finally, in the study published by GafterGvili [7], in which PET/CT was defined as helpful if it led either to true positives or true negatives, both fever and male gender were significant predictors of PET/CT usefulness.

A key limitation of this study is its retrospective design. As a result, the diagnosis and workup of FUO were not standardized before proceeding to PET/CT. Nevertheless, our study produced reliable data on the usefulness of PET/CT in the work-up of FUO. We collected a large group of patients while excluding all those that did not meet acceptable criteria. We adhered to strict methodology in how we defined useful PET/CT scans and discounted all PET/CT findings which were not diagnostic. With our standardized protocol, we identified in which patients PET/CT may be most valuable in assisting in the diagnosis of FUO.

\section{Conclusion}

In our study, PET/CT test was found to be highly beneficial in the work-up of FUO. It led to the diagnosis in nearly half of the patients in which a diagnosis could not be established following a thorough medical evaluation. Moreover, in patients with weight loss and anemia, PET/CT is most likely to be helpful in reaching the final diagnosis. Clinicians should be aware of the clinical utility of PET/CT and its place in the work-up of FUO following the completion of routine testing. 


\section{References}

1. Petersdorf, R. G. \& Beeson, P. B. (1961) Fever of unexplained origin: Report on 100 cases. Medicine , 40, 1-30. doi: 10.1097/00005792-196102000-00001

2. Bleeker-Rovers, C. P., Vos, F. J., de Kleijn, E. M., Mudde, A. H., Dofferhoff, T. S., Richter, C., ... van der Meer, J. W. (2007) A prospective multicenter study on fever of unknown origin: The yield of a structured diagnostic protocol. Medicine, 86, 26-38. doi: 10.1097/MD.0b013e31802fe858

3. Bharucha, T., Rutherford, A., Skeoch, S., Alavi, A., Brown, M., \& Galloway, J. (2017). Diagnostic yield of FDG-PET/CT in fever of unknown origin: a systematic review, meta-analysis, and Delphi exercise.Clin. Radiol . 72, 764-771. doi: 10.1016/j.crad.2017.04.014

4. Qiu, L. \& Chen, Y. (2012). The role of 18F-FDG PET or PET/CT in the detection of fever of unknown origin. Eur. J. Radiol. 81, 3524-3529. doi: 10.1016/j.ejrad.2012.05.025

5. Crouzet, J., Boudousq, V., Lechiche, C., Pouget, J. P., Kotzki, P. O., Collombier, L., .. Sotto, A. (2012). Place of (18)F-FDG-PET with computed tomography in the diagnostic algorithm of patients with fever of unknown origin. Eur. J. Clin . Microbiol. Infect. Dis. 31, 1727-33. doi: 10.1007/s10096-011-1440-6

6. Balink, H., Collins, J, Bruyn, G. \& Gemmel, F. (2009). F-18 FDG PET/CT in the diagnosis of fever of unknown origin. Clin. Nucl. Med. 34, 862-868. doi: 10.1097/RLU.0b013e3181becfb1

7. Gafter-Gvili, A., Raibman, S., Grossman, A., Avni, T., Paul, M., Leibovici, L., .. Bernstine, H. (2015). [18F]FDG-PET/CT for the diagnosis of patients with fever of unknown origin. QJM . 108, 289-298. doi: 10.1093/qjmed/hcu193

8. Schönau, V., Vogel, K., Englbrecht, M., Wacker, J., Schmidt, D., Manger, B., .. Schett, G. (2018). The value of 18F-FDG-PET/CT in identifying the cause of fever of unknown origin (FUO) and inflammation of unknown origin (IUO): data from a prospective study. Ann. Rheum. Dis. 77, 70-77. doi: 10.1136/annrheumdis2017-211687

9. Davidson, T., Kedmi, M., Avigdor, A., Komisar, O., Chikman, B, Lidar, M., ... Ben-Haim, S. (2018). FDG PET-CT evaluation in neurolymphomatosis: imaging characteristics and clinical outcomes. Leukemia \& Lymphoma 59, 348-356. doi: 10.1080/10428194.2017.1352096.

10. Davidson, T., Lotan, E., Klang, E., Nissan, J., Goldstein, J., Goshen, E., .. Chikman, B. (2018). Fat necrosis after abdominal surgery: A pitfall in interpretation of FDG-PET/CT. Eur Radiol. 28, 2264-2272. doi: $10.1007 / \mathrm{s} 00330-017-5201-5$

11. Davidson, T., Priel, E., Schiby, G., Raskin, S., Chikman, B., Nissan, E., .. Avigdor, A. (2018). Low rate of spleen involvement in sporadic Burkitt lymphoma at staging on PET-CT. Abdom Radiol. 43, 2369-2374 (2018).

12. Keidar, Z., Gurman-Balbir, A., Gaitini, D. \& Israel, O. (2008). Fever of unknown origin: The role of 18F-FDG PET/CT. J. Nucl. Med . 49, 1980-1985. doi: 10.2967/jnumed.108.054692

13. Federici, L., Blondet, C., Imperiale, A., Sibilia, J., Pasquali, J. L., Pflumio, F., ... Andres, E. (2010). Value of (18)F-FDG-PET/CT in patients with fever of unknown origin and unexplained prolonged inflammatory syndrome: a single centre analysis experience. Int. J. Clin. Pract . 64, 55-60. doi: 10.1111/j.17421241.2008.01774.x

14. Ferda, J., Ferdova, E., Zahlava, J., Matejovic, M. \& Kreuzberg, B. (2010). Fever of unknown origin: a value of (18)F-FDG-PET/CT with integrated full diagnostic isotropic CT imaging. Eur. J. Radiol . 73, 518-25. doi: 10.1016/j.ejrad.2008.12.014

15. Kei, P. L., Kok, T. Y., Padhy, A. K., Ng, D. C. \& Goh, A. S. (2010). [18F] FDG PET/CT in patients with fever of unknown origin: a local experience. Nucl. Med. Commun . 31, 788-92. doi: 10.1097/MNM.0b013e32833d0281. 
16. Sheng, J. F., Sheng, Z. K., Shen, X. M., Bi, S., Li, J. J., Sheng, G. P., .. Li, L. J. (2011). Diagnostic value of fluorine-18 fluorodeoxyglucose positron emission tomography/computed tomography in patients with fever of unknown origin. Eur. J. Intern. Med . 22, 112-116 (2011).

17. Pelosi, E., Skanjeti, A., Penna, D. \& Arena, V. Role of integrated PET/CT with [18F]-FDG in the management of patients with fever of unknown origin: a single-centre experience. Radiol. Med . 116, 809-20. doi: 10.1016/j.ejim.2010.09.015

18. Pedersen, T. I., Roed, C., Knudsen, L. S., Loft, A., Skinhoj, P., \& Nielsen, S. D. (2012). Fever of unknown origin: a retrospective study of 52 cases with evaluation of the diagnostic utility of FDG-PET/CT.Scand. J. Infect. Dis. 44, 18-23. doi: 10.3109/00365548.2011.603741

19. Kim, Y. J., Kim, S. I., Hong, K.-W. \& Kang, M. W. (2012). Diagnostic value of 18F-FDG PET/CT in patients with fever of unknown origin. Intern. Med . J . 42, 834-7. doi:10.1111/j.1445-5994.2012.02828.x

20. Pereira, A. M. V., Husmann, L., Sah, B.-R. R., Battegay, E. \& Franzen, D. (2016). Determinants of diagnostic performance of 18F-FDG PET/CT in patients with fever of unknown origin. Nucl. Med. Commun . 37, 57-65. doi: 10.1097/MNM.0000000000000395.

21. Hung, B. T., Wang, P. W., Su, Y. J., Huang, W. C., Chang, Y. H., Huang, S. H., \& Chang, C. C. (2017). The efficacy of $18 \mathrm{~F}-\mathrm{FDG} \mathrm{PET} / \mathrm{CT}$ and $67 \mathrm{Ga}$ SPECT/CT in diagnosing fever of unknown origin. Int. J. Infect. Dis. 62, 10-17. doi: 10.1016/j.ijid.2017.06.019

22. Buysschaert, I., Vanderschueren, S., Blockmans, D., Mortelmans, L., \& Knockaert, D. (2004). Contribution of (18)fluoro-deoxyglucose positron emission tomography to the work-up of patients with fever of unknown origin. Eur. J. Intern. Med . 15, 151-156. doi: 10.1016/j.ejim.2004.01.018

23. Ergul, N., Halac, M., Cermik, T. F., Ozaras, R., Sager, S., Onsel, C., \& Uslu, I. (2011). The Diagnostic Role of FDG PET/CT in Patients with Fever of Unknown Origin. Mol. Imaging Radionucl. Ther. 20, 19-25. doi: 10.4274/MIRT.20.04

24. Vanderschueren, S., Knockaert, D., Adriaenssens, T., Demey, W., Durnez, A., Blockmans, D., \& Bobbaers, H. (2003). From prolonged febrile illness to fever of unknown origin: The challenge continues. Arch. Intern. Med . 163, 1033-1041. doi: 10.1001/archinte.163.9.1033

\section{Conflict of interest Statement}

No specific funding was received from any bodies in the public, commercial or not-for-profit sectors to carry out the work described in this article.

The authors whose names are listed immediately below declare that there is no conflict of interest. They certify that they have NO affiliations with or involvement in any organization or entity with any financial interest (such as honoraria; educational grants; participation in speakers' bureaus; membership, employment, consultancies, stock ownership, or other equity interest; and expert testimony or patent-licensing arrangements), or non-financial interest (such as personal or professional relationships, affiliations, knowledge or beliefs) in the subject matter or materials discussed in this manuscript.

Authors: Hussein Mahajna, Keren Vaknin, Abdulla Watad, Arsalan Abu-Much, Naim Mahroum, Ora Shovman, Jennifer Ben Shimol, Yehuda Shoenfeld, Howard Amital, Tima Davidson

\section{Hosted file}

The utility of PET FUO Figures.docx available at https://authorea.com/users/353518/articles/ 477354-the-utility-of-pet-ct-in-diagnosing-fever-of-unknown-origin-the-experience-of-alarge-tertiary-medical-center

\section{Hosted file}


The utility of PET FUO Tables.docx available at https://authorea.com/users/353518/articles/ 477354-the-utility-of-pet-ct-in-diagnosing-fever-of-unknown-origin-the-experience-of-alarge-tertiary-medical-center 